\title{
Metabolomics of the effect of AMPK activation by AICAR on human umbilical vein endothelial cells
}

\author{
NURIA MARTÍNEZ-MARTÍN ${ }^{1}$, ANA BLAS-GARCÍA ${ }^{1,2}$, JOSE M. MORALES $^{3}$, \\ MIGUEL MARTI-CABRERA ${ }^{1}$, DANIEL MONLEÓN ${ }^{4 *}$ and NADEZDA APOSTOLOVA ${ }^{1 *}$ \\ ${ }^{1}$ Departamento de Farmacología, Facultad de Medicina, Universidad de Valencia-CIBERehd; ${ }^{2}$ Fundación \\ Hospital Universitario Dr Peset; ${ }^{3}$ Laboratorio de Imagen Molecular, Facultad de Medicina, Universidad \\ de Valencia; ${ }^{4}$ Fundación Hospital Clínico Universitario de Valencia-INCLIVA, Valencia, Spain
}

Received January 21, 2011; Accepted March 28, 2011

DOI: 10.3892/ijmm.2011.802

\begin{abstract}
AMP-activated protein kinase (AMPK) is a metabolic master switch expressed in a great number of cells and tissues. AMPK is thought to modulate the cellular response to different stresses that increase cellular AMP concentration. The adenosine analog, 5-aminoimidazole-4carboxamide-1- $\beta$-D-ribofuranoside (AICAR) is an AMPK activator used in many studies to assess the effects of AMPK activation on cellular metabolism and function. However, the effect of AICAR on cell metabolism reaches many different pathways and metabolites, some of which do not seem to be fully related to AMPK activation. We have now for the first time used NMR metabolomics on human umbilical vein endothelial cells (HUVEC) for the study of the global metabolic impact of AMPK activation by AICAR. In our study, incubation with AICAR activates AMPK and is associated with, among others, broad metabolic alterations in energy metabolism and phospholipid biosynthesis. Using NMR spectroscopy and metabolic network tools, we analyzed the connections between the different metabolic switches activated by AICAR. Our approach reveals a strong interconnection between different phospholipid precursors and oxidation by-products. Metabolomics profiling is a useful tool for detecting major metabolic alterations, generating new hypotheses and provides some insight about the different molecular correlations in a complex system. The present study shows that AICAR induces metabolic effects in cell metabolism well beyond energy production pathways.
\end{abstract}

Correspondence to: Dr Daniel Monleón, Fundación de Investigación del Hospital Clínico Universitario de Valencia, Avda. Blasco Ibáñez 17, Valencia 46010, Spain

E-mail: daniel.monleon@uv.es

${ }^{*}$ Contributed equally

Key words: NMR spectroscopy, AICAR, AMP-activated protein kinase, metabolic profiling

\section{Introduction}

AMP-activated protein kinase (AMPK) is a metabolic master switch expressed in a great variety of cells and tissues. AMPK regulates several intracellular systems including glucose cellular uptake, fatty acid $\beta$-oxidation and mitochondrial biogenesis (1-4). As a consequence, AMPK is thought to modulate the cellular response to different stresses that increase cellular AMP concentration (5-7). The adenosine analog, 5 -aminoimidazole-4-carboxamide-1- $\beta$-D-ribofuranoside (AICAR), is an AMPK activator used in many studies to assess the effects of this enzyme on cellular metabolism and function (8-10). AICAR activates AMPK without changing the levels of cellular adenine nucleotides. Thus, many studies link the activation of AMPK by AICAR with diverse processes, such as the transcription of glucose and fatty acid transport proteins (9), membrane expression of the creatine transporter (11), phosphorylation of key regulatory enzymes of lipid metabolism (12), and phosphatidylcholine and phosphatidylethanolamine biosynthesis (13). The metabolic impact of AMPK activation or inhibition is, therefore, very broad and highly complex. The analysis of metabolic profiles by metabolomic techniques may provide a global perspective about the different processes affected by AMPK activation and their possible interrelationships.

Nuclear magnetic resonance (NMR) is a non-invasive technique currently used in medical diagnosis and prognosis of human disease. High resolution magic angle spinning (HR-MAS) MR spectroscopy allows the determination of the metabolite content of semi-solid and viscous samples. Thus, for non-solid or highly viscous liquids, HR-MAS NMR spectroscopy allows the reduction of most of the line broadening associated with restricted molecular motion, chemical shift anisotropy, dipolar couplings and field inhomogeneity by high-rate spinning of the sample at the magic angle $\theta=54.7^{\circ}(14,15)$. The potential of HR-MAS applications to the study of cell cultures and biological tissues has been widely demonstrated in the investigation of different cellular alterations (16-18). In addition to the metabolites observed by conventional liquid NMR spectroscopy, HR-MAS provides information on the lipid content and other molecules that may be altered by extraction methods. The extraction process via 
protein precipitation methods disables the direct observation of, among others, membrane semi-mobile lipids. On the contrary, HR-MAS techniques provide high metabolic detail of unprocessed cells.

In the present study, we used HR-MAS spectroscopy on human umbilical vein endothelial cells (HUVEC) for the metabolomics study of the global metabolic impact of AMPK activation by AICAR. As the major regulator of vascular homeostasis, the endothelium maintains the balance between vasodilation and vasoconstriction, inhibition and stimulation of smooth muscle cell proliferation and migration, and thrombogenesis and fibrinolysis. When this balance is upset, endothelial dysfunction occurs, which is considered an early marker for atherosclerosis. Many pathological cardiovascular conditions such as myocardial infarction, hypertension, and diabetes have been shown to increase cellular stresses that can activate AMPK, producing direct and indirect modulatory effects on vascular endothelium function. In fact, there is growing evidence of a vasculoprotective role of AMPK in this tissue. Understanding the metabolic changes induced by AMPK activation could help the development of AMPK as a therapeutic target for cardiovascular disease (19).

We report that incubation with AICAR activates AMPK and is associated with broad metabolic alterations in energy metabolism and phospholipid synthesis, among others.

\section{Materials and methods}

HUVEC culture. HUVEC were obtained from fresh human umbilical cords (Department of Gynecology, Hospital Clínico Universitario, Valencia, Spain) and isolated by extraction with collagenase (20). Cells were cultured according to the manufacturer's instructions in EMG-2 medium supplemented with BulletKit components (Clonetics ${ }^{\mathrm{TM}}$, Lonza, Walkersville, MD) and 50 units $/ \mathrm{ml}$ penicillin, $50 \mu \mathrm{g} / \mathrm{ml}$ streptomycin and $2.5 \mu \mathrm{g} /$ $\mathrm{ml}$ fungizone (amphotericin B). HUVEC were maintained in an incubator (IGO 150, Jouan, Saint-Herblain Cedex, France) at $37^{\circ} \mathrm{C}$ and in a humidified atmosphere of $5 \% \mathrm{CO}_{2} / 95 \%$ air (AirLiquide). All protocols complied with European Community guidelines for the use of human experimental models and were approved by the Ethics Committee of the University of Valencia.

HR-MAS spectroscopy. At least 3 different sets of samples were studied by NMR for reproducibility check purposes. Cell cultures were centrifuged for $5 \mathrm{~min}$ at $5000 \mathrm{rpm}$. Of the resulting pellet, $40 \mu \mathrm{l}$ were introduced in a $4 \mathrm{~mm} \mathrm{ZrO}_{2}$ rotor fitted with a $50 \mathrm{ml}$ cylindrical insert. Some $\mathrm{D}_{2} \mathrm{O}$ was added to the sample for field locking purposes. Then, the rotor was transferred into the NMR probe, which had been previously cooled at $10^{\circ} \mathrm{C}$ for minimizing sample degradation. The whole HR-MAS study was performed at this temperature, and it was initiated immediately after the temperature inside the probe reached the equilibrium condition $(5 \mathrm{~min})$. The HR-MAS spectrum was recorded on a Bruker Avance- 600 spectrometer operating at a frequency of $600.13 \mathrm{MHz}$. The instrument was equipped with a $4 \mathrm{~mm}$ triple resonance HR-MAS probe. A Bruker cooling unit was used to control the temperature by cooling down the bearing air flowing into the probe. The sample was spun at $5000 \mathrm{~Hz}$ in order to keep the rotation sidebands out of the acquisition window. One-dimensional proton spectrum with water pre-saturation was acquired in $15 \mathrm{~min}$. The data was then processed using the spectrometer software Topspin 1.3 (Bruker Biospin GmbH, Germany). The spectral vector was then transferred to MATLAB for additional processing and further analysis. The peak areas were calculated by deconvolution of the region of interest with the in-house MATLAB software. Peaks were fitted to a Voight-shape peak and the calculated area was normalized to the global spectral intensity.

Statistical analysis. Values are the mean \pm standard error of the mean (SEM) of 3 experiments. Statistical analysis was performed by one-way ANOVA followed by a Student Newman-Keuls test for unpaired samples (MATLAB, MathWorks Inc., 2008). P-values of $<0.05$ and $<0.01$ indicate significant differences.

Graphical network analysis. Biochemical reactions involving the identified metabolites were identified through pathways annotated in the Kyoto Encyclopedia of Genes and Genomes (KEGG) (21) with a super-network created from all pathways which involved 3 or more of the metabolites identified here. Graphical network analysis was conducted with Cytoscape v. 2.3.1 (http://www.cytoscape.org) and its plug-in MetScape 1.0.1 (http://metscape.ncibi.org). Two types of networks were generated for visualization, pathway-ordered and degreeordered. Each metabolite in the network is represented as a node and each chemical reaction connecting two metabolites is represented as an edge connecting both boxes. In the pathway-ordered network visualization, network nodes are treated like physical objects that repel each other, such as electrons. The connections between the nodes are treated like metal springs attached to the pair of nodes. These springs repel or attract their end points according to a force function. The layout algorithm sets the positions of the nodes in a way that minimizes the sum of forces in the network. In the degree-ordered network, all of the nodes in the network are placed around a circle. Each node is circularly sorted based on the total number of edges in which it participates. The result is that all nodes involved in the same number of chemical reactions are located together around the circle.

\section{Results}

We performed high resolution NMR spectroscopy on HUVEC cells incubated with and without AICAR for $3 \mathrm{~h}$ to assess the global metabolic impact. The resulting NMR spectra showed narrow line widths and adequate signal-to-noise ratios with well-resolved spin-spin multiplicities, as shown in Fig. 1. Metabolite spin systems and resonances were identified by using literature data (22) and additional ${ }^{2} \mathrm{D}$ homo- and multinuclear experiments collected in selected samples. The ${ }^{1} \mathrm{D}$ single-pulse pre-saturation experiment provides complete and unambiguous identification of the metabolic pattern characterizing the examined cells. The most relevant metabolites are labeled in Fig. 1. In all spectra, the aliphatic region had dominant fatty acids signals. These signals arise from saturated fatty acids moieties $\left(-\mathrm{CH}_{2} \underline{\mathrm{CH}}_{2} \mathrm{CH}_{2}-\right.$ at $1.3 \mathrm{ppm}$ and $-\mathrm{CH}_{2} \underline{\mathrm{CH}}_{3}$ at $0.9 \mathrm{ppm})$ and unsaturated fatty acids moieties $(-\mathrm{CH}=\underline{\mathrm{CH}}-$ at 

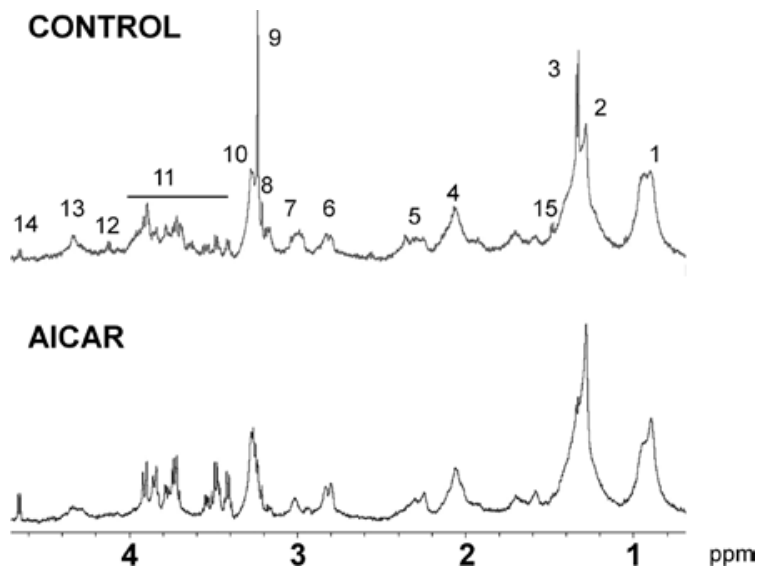

Figure 1. Aliphatic region of single pulse ${ }^{1} \mathrm{H}$ HR-MAS NMR water suppressed spectra of $40 \mu 1$ of the HUVEC pellet measured at $10^{\circ} \mathrm{C}$ with (bottom) and without (top) AICAR. The labels indicate the spectral position of the most significant metabolites (see text for details). Key: $1, \mathrm{CH}_{3}-\left(\mathrm{CH}_{2}\right) \mathrm{n}$ lipids; 2, $\left(\mathrm{CH}_{2}\right)$ n lipids; $3, \mathrm{CH}_{3}$ lactate; $4, \mathrm{CH}=\mathrm{CH}-\mathrm{CH}_{2}-\mathrm{CH}_{2}$ lipids; $5, \mathrm{CH}_{2-}$ $\mathrm{CH}_{2}-\mathrm{CO}$ lipids; $6,=\mathrm{CH}-\mathrm{CH}_{2}-\mathrm{CH}=$ lipids; 7 , creatine and phsophocreatine; 8, free choline, 9; phosphocholine; 10, taurine, betaine, choline-containing compounds and ethanol-amine-containing compounds; 11, peaks belonging to amino acids and other small metabolites (glucose, creatine, inositols, glycerol); 12, CH lactate; 13, phosphocholine; 14, glucose.

$5.4 \mathrm{ppm}$ and $\mathrm{CH}=\mathrm{CH}-\mathrm{CH}_{2}$ at $\left.2.0 \mathrm{ppm}\right)$. The spectra also showed prominent signals of water-soluble metabolites such as lactate, $\alpha$ - and $\beta$-glucose, creatine and taurine. Signals belonging to phospholipids also had strong intensities at $3.2 \mathrm{ppm}$. The high chemical shift resolution obtained by MAS at $14.1 \mathrm{~T}$ allowed to assign individual signals from membrane phospholipids compounds including Choline (Cho), phosphocholine (PCho), ethanolamine (EA) and phosphoethanolamine (PEA). The endogenous compounds detected in the spectra also included standard amino acids like glycine, leucine, isoleucine, valine, alanine, lysine, asparagine, aspartic acid, glutamine and glutamate.

The metabolic changes induced by AICAR treatment and detected by NMR spectroscopy in HUVEC cultures are highly reproducible. These reproducible changes were quantified by calculating the relative spectra peak area ratios (Table I and Fig. 2). The most prominent changes include strong decreases in the intensity of signals of phospholipids compounds. These decreases are most important for PCho at $3.20 \mathrm{ppm}$, Cho at $3.19 \mathrm{ppm}$ and PEA at $3.23 \mathrm{ppm}$. Additionally, and in the same spectral region, there is a large increase in the signal intensities of betaine protons (3.22 and $3.89 \mathrm{ppm}$ ). Other interesting changes in the spectral pattern of HUVEC induced by treatment with AICAR include the signal intensity increases from the glucose moieties (mainly that at $4.65 \mathrm{ppm}$ ) and the decrease from lactate methyl (at 1.33 and $4.11 \mathrm{ppm}$ ). In contrast to these changes, an increase in the levels of saturated fatty acids at 1.30 and $0.94 \mathrm{ppm}$ and mono-unsaturated and poly-unsaturated fatty acids (i.e. PUFA bisallylic methylene fatty acyl protons, at $2.79 \mathrm{ppm}$ ) suggests some alteration of the energy production-consumption pathways. The analysis of relaxation-filtered spectra and diffusion-weighted spectra performed on these samples suggests that the major contribution to these resonances comes from mobile lipids in large lipid droplets (23). Signals belonging to the moieties of glutamine, glutamate and aspartic acid appear to be increased in the cells treated with AICAR. On the other hand, levels of

Table I. Effect of AICAR on the metabolic profile of HUVEC.

\begin{tabular}{|c|c|c|c|c|}
\hline & $\begin{array}{c}\text { Control } \\
\text { (a.u.) }\end{array}$ & $\begin{array}{c}\text { AICAR } \\
\text { (a.u.) }\end{array}$ & $\begin{array}{c}\text { Change } \\
(\%)\end{array}$ & P-value \\
\hline \multicolumn{5}{|l|}{ Fatty Acids } \\
\hline$-\mathrm{CH}_{2} \mathrm{CH}_{3}(1)$ & $1040 \pm 190$ & $1320 \pm 120$ & $125 \pm 4$ & 0.039 \\
\hline$-\mathrm{CH}_{2} \mathrm{CH}_{2} \mathrm{CH}_{2^{-}}(2)$ & $1370 \pm 210$ & $2010 \pm 250$ & $141 \pm 8$ & 0.039 \\
\hline$-\mathrm{CH}=\mathrm{CHCH}_{2}-(4)$ & $540 \pm 180$ & $630 \pm 210$ & $115 \pm 5$ & 0.762 \\
\hline$-\mathrm{CH}_{2} \mathrm{CH}_{2}-\mathrm{CO}(5)$ & $320 \pm 140$ & $370 \pm 160$ & $116 \pm 4$ & 0.865 \\
\hline$=\mathrm{CH}-\mathrm{CH}_{2}-\mathrm{CH}=(6)$ & $370 \pm 60$ & $480 \pm 40$ & $127 \pm 4$ & 0.108 \\
\hline Lactate $(3,12)$ & $810 \pm 130$ & $540 \pm 50$ & $65 \pm 6$ & 0.031 \\
\hline Creatine/phosphocreatine (7) & $350 \pm 130$ & $180 \pm 60$ & $52 \pm 10$ & 0.889 \\
\hline Cho $(8)$ & $43 \pm 7$ & $25 \pm 4$ & $58 \pm 7$ & 0.051 \\
\hline PCho $(9,13)$ & $165 \pm 37$ & $31 \pm 4$ & $17 \pm 3$ & 0.005 \\
\hline PEA (10) & $24 \pm 6$ & $12 \pm 6$ & $55 \pm 10$ & 0.067 \\
\hline EA (10) & $26 \pm 5$ & $7 \pm 2$ & $26 \pm 5$ & 0.009 \\
\hline Betaine (10) & $11 \pm 3$ & $26 \pm 4$ & $32 \pm 6$ & 0.016 \\
\hline Glucose (14) & $13 \pm 4$ & $31 \pm 9$ & $322 \pm 42$ & 0.008 \\
\hline Alanine (15) & $27 \pm 9$ & $12 \pm 5$ & $44 \pm 13$ & 0.009 \\
\hline
\end{tabular}

Relative NMR peak area ratios from single pulse ${ }^{1} \mathrm{H}$ water-suppressed spectra for metabolites showing most consistent changes with AICAR treatment. Significant P-values are indicated in bold. The peak number(s) used for the quantitation are shown in parentheses. The results are expressed as means \pm standard error from three separate HUVEC preparations. Cho, choline; PCho, phosphocholine; EA, ethanolamine; PEA, phosphoethanolamine. 

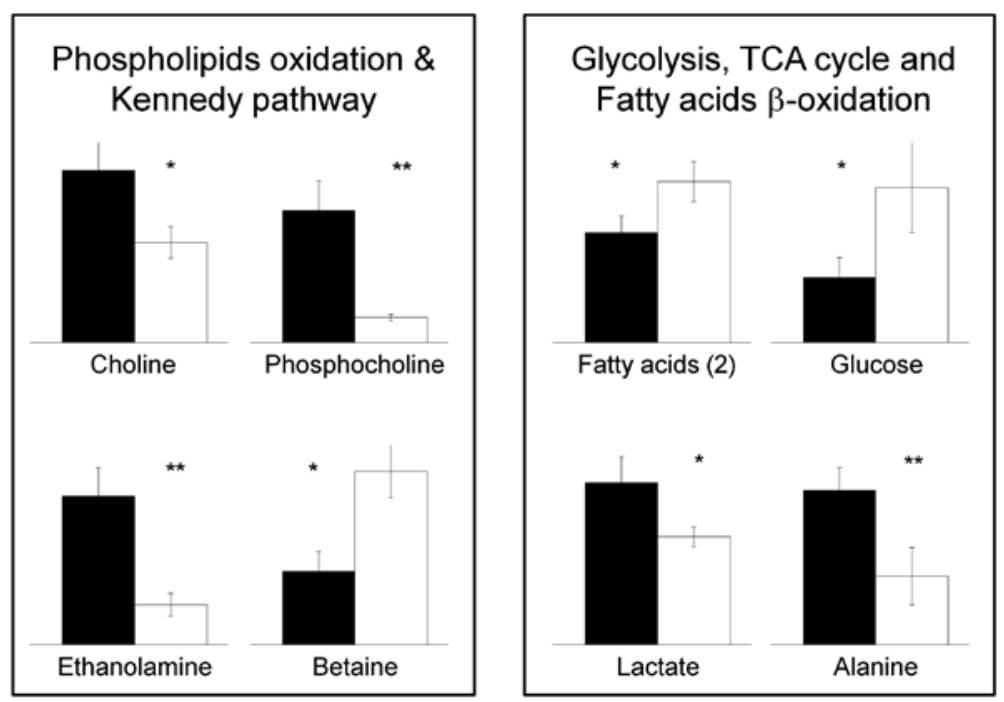

Figure 2. Histograms showing the metabolite levels for HUVEC cells incubated without (black) and with (white) AICAR. Results are expressed as means \pm standard errors. ${ }^{*} \mathrm{P}<0.05 ;{ }^{* *} \mathrm{P}<0.01$.

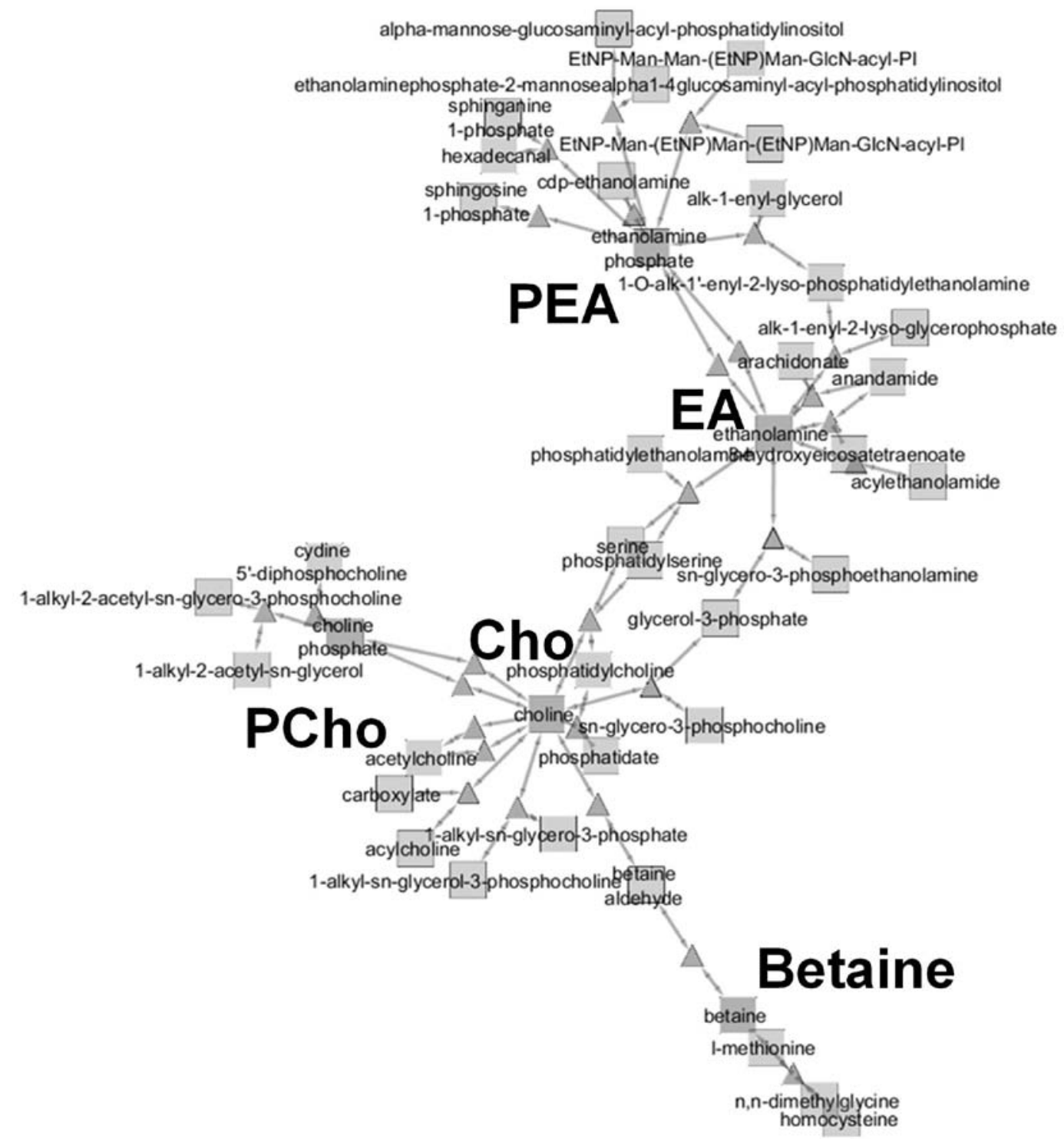

Figure 3. Spring-embedded visualization of the phopholipid metabolism cluster from the metabolic profile of the AMPK activation in HUVEC cells by incubation with AICAR. The pathways were derived from KEGG and the figure was generated with MetScape and Cytoscape. 


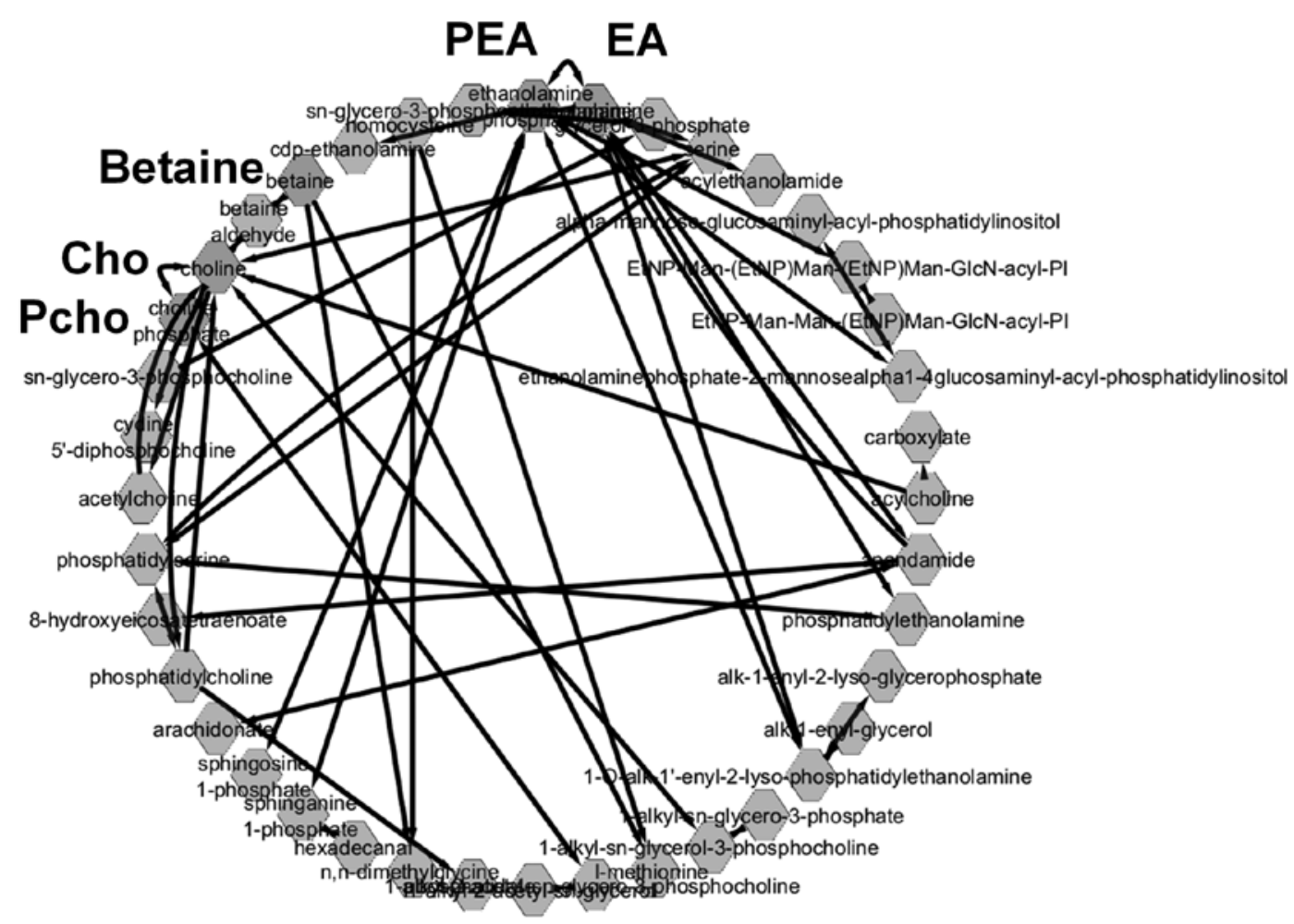

Figure 4. Degree-ordered network analysis of the phospholipid metabolism cluster of the metabolic profile of the AMPK activation in HUVEC cells by incubation with AICAR. The pathways were derived from KEGG and the figure was generated with MetScape and Cytoscape.

alanine, leucine, isoleucine and valine are lower in these cells in comparison to the control cells.

A network pathway analysis was conducted in order to better understand the possible connections of these metabolic alterations and to complement traditional pathway analysis via KEGG pathways. Our analysis showed that most AICARinduced metabolic alterations involved two major clusters corresponding to energy production pathways and phospholipid metabolism. Within these clusters, Figs. 3 and 4 demonstrate that a majority of the metabolites identified were connected by a maximum of three chemical reactions. The number of potential pathways involved highlights the complexity of the metabolic response to the AMPK activation by AICAR.

\section{Discussion}

Metabolomics is the systematic study of the unique chemical fingerprints that specific cellular processes leave behind (24). AMPK activation induces many different metabolic changes in the cell. The use of metabolomics for the study of such systems provides unique opportunities to detect relevant metabolic patterns and complex interactions. In the present study, we have used NMR metabolomics for the first time to obtain a global view of the metabolic impact of AMPK activation by AICAR. Each NMR spectra provided quantitative information about many metabolites allowing the detection, not only of individual metabolic changes (Fig. 2) but also potential metabolic connections between the pathways affected (Figs. 3 and 4).

Activation of AMPK by AICAR drastically reduces the signals of PCho in the spectra. PCho is an intermediate in the synthesis of phosphatidylcholine in tissues and is produced in a reaction, catalyzed by choline kinase, which converts ATP and Cho into PCho and ADP. This result is consistent with previous studies which showed that AICAR inhibits synthesis of PCho and PEA and activates the oxidation of Cho to betaine in hepatocytes (13). The increase in the broad signal intensities of betaine protons (3.22 and $3.89 \mathrm{ppm}$ ) seems to confirm this effect. Betaine NMR signal includes any neutral chemical compound with a positively charged cationic functional group such as a quaternary ammonium or a phosphonium cation. The contribution of many chemically similar molecules to this signal broadens the betaine peak more than expected. Many betaines serve as organic osmolytes for protection against osmotic stress, drought, high salinity or high temperature. Intracellular accumulation of betaines permits water retention in cells, thus protecting them from the effects of dehydration. These metabolic effects may partially explain recent studies reporting that metabolic preconditioning with AICAR increases freeze tolerance of several cell lines and primary rat hepatocytes (25).

Metabolic changes involving glucose, lactate and fatty acids partially support the hypothesis that AICAR decreases glycolysis and increases fatty acid oxidation in HUVEC by activation of AMPK (26). Glucose signals in the NMR spectra are higher after AICAR incubation. Most of these signals include contribution from phosphorylated glucose. When glucose is transported into the cell it is phosphorylated by hexokinase. This phosphorylation keeps glucose from leaving the cell, and by changing the structure of glucose through phosphorylation, it decreases the concentration of glucose molecules, resulting in a gradient which allows for more 
glucose to be transported into the cell. The hexokinase II transcription is increased in both red and white skeletal muscle upon treatment with AICAR (27). On the other hand, AICAR treatment has been shown to increase the expression of the glucose transporter type 4 (GLUT4) (28). Collectively, the data suggest that the increase in the glucose signals responds to a decreased glycolysis rate combined with a higher glucose uptake.

AICAR treatment in endothelial cells also produces an increase in the signal of fatty acids, which stems mainly from accumulation of mobile lipids. The increase in NMR visible fatty acids can result either from increased lipolysis or from increased production. In our system, changes in mobile lipids are probably the outcome of AMPK activation, which controls several fatty acid-related pathways, such as lipid uptake, fatty acid oxidation or fatty acid synthesis. As many pathological cardiovascular conditions increase stresses that could activate AMPK, it is plausible to speculate that the activation of this enzyme could exert beneficial effects in these cells, reducing lipid accumulation to produce ATP and stimulating enzymes responsible for nitric oxide formation (29). However, in sustained pathological conditions involving an impairment of the oxidative mitochondrial pathway, this response could lead to lipid accumulation and may be involved in processes such as endothelial dysfunction or the initial phases of atherosclerotic disease.

AMPK is a metabolic energy switch activated when some type of cellular stress depletes ATP. The resulting increased AMP/ATP ratio activates AMPK, which in turn induces the inhibition of many energy-consuming metabolic pathways and the activation of energy producing pathways. As part of this scenario, recent studies suggest that AMPK achieves these effects by coupling cell metabolism and membrane transport (30). The creatine kinase (CK)-phosphocreatine system is important for tissues and cells with rapid and variable energy demand (31). Phosphocreatine is an important energy reservoir, which may rapidly release ATP under certain conditions. In this study, we observed that AICAR induces a decrease of intracellular total creatine (phosphocreatine plus creatine). This suggests a decrease in creatine transport. Along these lines, Li et al recently showed that AICAR inhibits $\mathrm{Na}^{+}$-dependent creatine uptake via the creatine transporter (CRT) into oocytes (11). The reasons for this inhibition and the overall role of AMPK as metabolic response to stress are unclear. However, creatine transport for intracellular energy storage may be energetically wasteful, as it would tend to dissipate cellular ionic gradients, through the concomitant pumping of $\mathrm{Na}^{+}$, and would require a higher $\mathrm{Na}^{+}-\mathrm{K}^{+}$-ATPase activity. Further studies are needed to elucidate the role of AMPK in the transport and usage of creatine.

In summary, for the first time, we report a metabolomics approach to the study of metabolic alterations produced by AICAR-induced AMPK activation. The power of this approach to the study of systems with highly complex metabolic interactions is also demonstrated. By using NMR spectroscopy and metabolic network tools, we analyzed the connections between the different metabolic switches activated by AICAR. Our results suggest that AICAR has major effects on two main metabolic clusters involving energy production and transport and phospholipid biosynthesis. Some of these results were previously detected in independent studies, but this is the first time that they are detected simultaneously. Our approach also revealed a strong interconnection between different phospholipid precursors and oxidation by-products. Overall, our study suggests that global metabolic profiling is a useful tool for detecting major metabolic alterations, generating new hypotheses and may provide some insight into the different molecular correlations in a complex system.

\section{Acknowledgements}

This study was partly financed by grants from the Consellería de Sanidad (GV-AP064/07, GV-AP076/08, GV-AP014/09 and GV-AP023/10) and the Consellería de Educación (ACOMP2009-200, ACOMP2010-184, ACOMP2011-237, PROMETEO/2010/060 and FPA/2011/054) de la Generalitat Valenciana, the Ministerio de Educación y Ciencia del Gobierno de España (SAF2008-00270). D.M. gratefully acknowledges the Ministerio de Educación y Ciencia del Gobierno de España for a Ramon y Cajal 2006 Contract.

\section{References}

1. Winder WW and Hardie DG: AMP-activated protein kinase, a metabolic master switch: possible roles in type 2 diabetes. Am J Physiol 277: E1-E10, 1999.

2. Hallows KR, Alzamora R, Li H, et al: AMP-activated protein kinase inhibits alkaline $\mathrm{pH}$ - and PKA-induced apical vacuolar $\mathrm{H}^{+}$-ATPase accumulation in epididymal clear cells. Am J Physiol Cell Physiol 296: C672-C681, 2009.

3. Hardie DG, Hawley SA and Scott JW: AMP-activated protein kinase: development of the energy sensor concept. J Physiol 574: $7-15,2006$

4. Bergeron R, Ren JM, Cadman KS, et al: Chronic activation of AMP kinase results in NRF-1 activation and mitochondrial biogenesis. Am J Physiol Endocrinol Metab 281: E1340-E1346, 2001.

5. Viollet B, Horman S, Leclerc J, et al: AMPK inhibition in health and disease. Crit Rev Biochem Mol Biol 45: 276-295, 2010.

6. Lim CT, Kola B and Korbonits M: AMPK as a mediator of hormonal signalling. J Mol Endocrinol 44: 87-97, 2010.

7. Li C and Keaney JF Jr: AMP-activated protein kinase: a stressresponsive kinase with implications for cardiovascular disease. Curr Opin Pharmacol 10: 111-115, 2010.

8. Corton JM, Gillespie JG, Hawley SA and Hardie DG: 5-Aminoimidazole-4-carboxamide ribonucleoside. A specific method for activating AMP-activated protein kinase in intact cells? Eur J Biochem 229: 558-565, 1995.

9. Lemieux K,Konrad D, Klip A and Marette A: The AMP-activated protein kinase activator AICAR does not induce GLUT4 translocation to transverse tubules but stimulates glucose uptake and p38 mitogen-activated protein kinases alpha and beta in skeletal muscle. FASEB J 17: 1658-1665, 2003.

10. Kim JE, Kim YW, Lee IK, Kim JY, Kang YJ and Park SY: AMP-activated protein kinase activation by 5-aminoimidazole4-carboxamide-1-beta-D-ribofuranoside (AICAR) inhibits palmitate-induced endothelial cell apoptosis through reactive oxygen species suppression. J Pharmacol Sci 106: 394-403, 2008.

11. Li H, Thali RF, Smolak C, et al: Regulation of the creatine transporter by AMP-activated protein kinase in kidney epithelial cells. Am J Physiol Renal Physiol 299: F167-F177, 2010.

12. Hardie DG and Carling D: The AMP-activated protein kinase: fuel gauge of the mammalian cell? Eur J Biochem 246: 259-273, 1997.

13. Houweling M, Klein W and Geelen MJ: Regulation of phosphatidylcholine and phosphatidylethanolamine synthesis in rat hepatocytes by 5-aminoimidazole-4-carboxamide ribonucleoside (AICAR). Biochem J 362: 97-104, 2002.

14. Andrew ER and Eades RG: Removal of dipolar broadening of NMR spectra of solids by specimen rotation. Nature 183 : $1802-1803,1959$. 
15. Garroway AN: Magic-angle sample spinning of liquids. J Magn Reson 49: 168-171, 1982.

16. Weybright P, Millis K, Campbell N, Cory DG and Singer S: Gradient, high-resolution, magic angle spinning $1 \mathrm{H}$ nuclear magnetic resonance spectroscopy of intact cells. Magn Reson Med 39: 337-345, 1998.

17. Griffin JL, Bollard M, Nicholson JK and Bhakoo K: Spectral profiles of cultured neuronal and glial cells derived from HRMAS (1)H NMR spectroscopy. NMR Biomed 15: 375-384, 2002.

18. Monleon D, Morales JM, Gonzalez-Darder J, et al: Benign and atypical meningioma metabolic signatures by high-resolution magic-angle spinning molecular profiling. J Proteome Res 7: 2882-2888, 2008.

19. Xu Q and Si LY: Protective effects of AMP-activated protein kinase in the cardiovascular system. J Cell Mol Med 14: 2604-2613, 2010.

20. Jaffe EA, Nachman RL, Becker CG and Minick CR: Culture of human endothelial cells derived from umbilical veins. Identification by morphologic and immunologic criteria. J Clin Invest 52: 2745-2756, 1973.

21. Kanehisa M and Goto S: KEGG: Kyoto encyclopedia of genes and genomes. Nucleic Acids Res 28: 27-30, 2000.

22. Nicholson JK, Foxall PJ, Spraul M, Farrant RD and Lindon JC: $750 \mathrm{MHz}{ }^{1} \mathrm{H}$ and ${ }^{1} \mathrm{H}-{ }^{13} \mathrm{C}$ NMR spectroscopy of human blood plasma. Anal Chem 67: 793-811, 1995.

23. Barba I, Cabanas ME and Arus C: The relationship between nuclear magnetic resonance-visible lipids, lipid droplets, and cell proliferation in cultured C6 cells. Cancer Res 59: 1861-1868, 1999.
24. Nicholson JK, Lindon JC and Holmes E: 'Metabonomics': understanding the metabolic responses of living systems to pathophysiological stimuli via multivariate statistical analysis of biological NMR spectroscopic data. Xenobiotica 29: 1181-1189, 1999.

25. Menze MA, Chakraborty N, Clavenna M, et al: Metabolic preconditioning of cells with AICAR-riboside: improved cryopreservation and cell-type specific impacts on energetics and proliferation. Cryobiology 61: 79-88, 2010.

26. Dagher Z, Ruderman N, Tornheim K and Ido Y: The effect of AMP-activated protein kinase and its activator AICAR on the metabolism of human umbilical vein endothelial cells. Biochem Biophys Res Commun 265: 112-115, 1999.

27. Ojuka EO, Nolte LA and Holloszy JO: Increased expression of GLUT-4 and hexokinase in rat epitrochlearis muscles exposed to AICAR in vitro. J Appl Physiol 88: 1072-1075, 2000.

28. Merrill GF, Kurth EJ, Hardie DG and Winder WW: AICA riboside increases AMP-activated protein kinase, fatty acid oxidation, and glucose uptake in rat muscle. Am J Physiol 273: E1107-E1112, 1997.

29. Ewart MA and Kennedy S: AMPK and vasculoprotection. Pharmacol Ther: November 25, 2010 (Epub ahead of print).

30. Hallows KR: Emerging role of AMP-activated protein kinase in coupling membrane transport to cellular metabolism. Curr Opin Nephrol Hypertens 14: 464-471, 2005.

31. Neumann D, Schlattner U and Wallimann T: A molecular approach to the concerted action of kinases involved in energy homoeostasis. Biochem Soc Trans 31: 169-174, 2003. 